\title{
Variabilidade física e anatômica da madeira de Khaya ivorensis A. Chev. em diferentes espaçamentos de plantio
}

\author{
Physical and anatomical variability of Khaya ivorensis \\ A. Chev wood . in different planting spacings
}

\begin{abstract}
Denise Ransolin Soranso', Graziela Baptista Vidaurre², José Tarcísio da Silva Oliveira3, Mário Tomazello Filho ${ }^{4}$, João Gabriel Missia da Silva ${ }^{1}$ e Marina Donaria Chaves Arantes ${ }^{2}$
\end{abstract}

\begin{abstract}
Resumo
O objetivo deste estudo foi avaliar a influência do espaçamento entre árvores no crescimento radial e qualidade do lenho de árvores de Khaya ivorensis, aos 5 anos de idade. Os plantios estão localizados

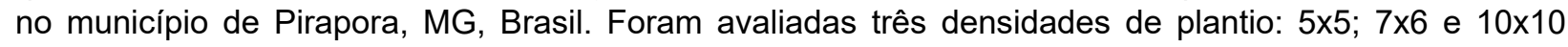
metros. Em cada espaçamento foram selecionadas 10 árvores considerando as direções cardiais norte e sul para remoção de amostras radiais do lenho, através de método não destrutivo, a $40 \mathrm{~cm}$ de altura do solo. Avaliou-se a influência do espaçamento de plantio na variação radial da densidade aparente da madeira, através da densitometria de raios $\mathrm{X}$, e das dimensões das fibras a partir de amostras do cerne periférico das baguetas da direção norte. Os dados foram submetidos à análise de variância, pelo teste $F$ e as médias comparadas pelo teste de Tukey, quando a hipótese da anuidade era rejeitada. Houve maior crescimento em diâmetro e incremento médio anual em diâmetro (IMAD) para as árvores plantadas nos maiores espaçamentos ( $7 \times 6$ e 10×10 m). A densidade aparente média e dimensões das fibras da madeira de $K$. ivorensis não diferiram entre si em função do espaçamento de plantio aos cinco anos de idade. A densidade aparente da madeira teve correlação significativa com o DAP das árvores plantadas, no maior espaçamento (10x10 m), tendendo esta a diminuir conforme aumento do diâmetro a altura do peito (DAP) das árvores. Em todos os espaçamentos houve tendência comum de variação radial da densidade aparente da madeira, sendo menor próximo à medula e conforme o distanciamento da mesma tendeu ao aumento próximo a casca.
\end{abstract}

Palavras-chave: mogno africano, crescimento em diâmetro, densidade da madeira.

\begin{abstract}
The objective of this study was to evaluate the influence of spacing between trees in radial growth and quality of Khaya ivorensis wood, at 5 years of age. The plantations are located in the city of Pirapora, MG,

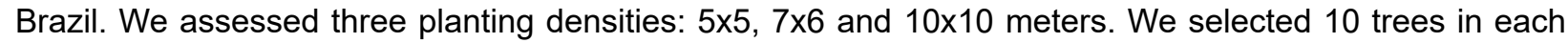
spacing considering the north and south cardinal directions to remove the radial wood samples using nondestructive method, at $40 \mathrm{~cm}$ above the ground. We evaluated the influence of planting spacing in the radial variation of the apparent density of the wood through X-ray densitometry, and the size of the fibers from the peripheral heartwood of the north radial samples. Data were subjected to analysis of variance by $F$ test and the means were compared by Tukey's test, when the null hypothesis was rejected. There was a higher diameter growth and mean annual increment (MAl) in diameter for the trees planted in greater spacings (7x6 and 10x10 m). The apparent average density and dimensions of $K$. ivorensis wood fibers did not differ with planting spacings at five years of age. The bulk density of the wood had significant correlation with $\mathrm{DBH}$ of trees planted in the greatest spacing $(10 \times 10 \mathrm{~m})$, tending to decrease as the DBH of trees increase. In all spacings, there was a common trend of radial variation of the apparent density of the wood, being lower near the core and increasing as the distance from the same tended to increase, next to the bark.
\end{abstract}

Keywords: African mahogany, diameter growth, wood density.

1Doutorando(a) em Ciências Florestais. UFES - Universidade Federal do Espírito Santo / Centro de Ciências Agrárias. Avenida Governador Lindemberg, 31 - Centro - 29550000 - Jerônimo Monteiro, ES, Brasil. E-mail: denise soranso@hotmail. com; j.gabrielmissia@hotmail.com.

2Professor(a) Adjunto no Departamento de Ciências Florestais e da Madeira. UFES - Universidade Federal do Espírito Santo, Centro de Ciências Agrárias. Av. Governador Lindemberg, 31 Centro - 29550000 - Jerônimo Monteiro, ES, Brasil. E-mail: grazividaurre@gmail.com; mdonariac@hotmail.com.

3Professor Associado no Departamento de Ciências Florestais e da Madeira. UFES - Universidade Federal do Espírito Santo, Centro de Ciências Agrárias. Av. Governador Lindemberg, 31 Centro - 29550000 - Jerônimo Monteiro, ES, Brasil. E-mail: jtsilva@npd.ufes.br

${ }^{4}$ Professor Titular do Departamento de Ciências Florestais. USP - Universidade de São Paulo / ESALQ - Escola Superior de Agricultura Luiz de Queiroz. Av. Pádua Dias, 11 - 13418900 - Piracicaba, SP, Brasil. E-mal: mtomazel@usp.br.

Sci. For., Piracicaba, v. 44, n. 110, p. 519-526, jun. 2016

DOI: dx.doi.org/10.18671/scifor.v44n110.24 


\section{INTRODUÇÃO}

Para o sucesso na implantação de um povoamento florestal, a adoção de práticas silviculturais adequadas é uma ação importante, para o desenvolvimento de uma relação direta entre produtividade e qualidade da matéria prima da qual será destinado o plantio.

A definição do espaçamento inicial é uma das decisões mais importantes na implantação de florestas, pois proporciona para cada indivíduo o espaço suficiente para se obter o crescimento máximo, exercendo influência nos demais tratamentos culturais, na qualidade da madeira, na colheita florestal e, consequentemente, nos custos de produção (SIMÕES et al., 1976; LEITE et al., 2006).

Espécies exóticas como Khaya ivorensis, conhecida popularmente como Mogno Africano, têm se destacado pelo seu crescente cultivo no Brasil, apresentando rápido crescimento e fácil adaptação. É uma espécie de origem africana, produtora de madeira com características tecnológicas desejada para produção de madeira sólida destinada a serraria.

No entanto, existem alguns questionamentos sobre o espaçamento inicial ideal para a formação de plantios de K. ivorensis no Brasil. Sabe-se que os plantios comerciais existentes da espécie estão em fase inicial de desenvolvimento, os quais empregam espaçamentos amplos $(4 \times 5 ; 5 \times 5 ; 6 \times 6 ; 7 \times 6$ $\mathrm{m}$ ), como uma estratégia para que não sejam realizados desbastes futuros no plantio (FERRAZ; RIBEIRO, 2013).

Todavia, é importante que estudos sejam realizados nas fases iniciais de estabelecimento dessas florestas, verificando como a espécie vem se desenvolvendo em relação às condições de plantio em que foram submetidas, com a definição de práticas silviculturais adequadas visando a produção de madeira com a qualidade e crescimento desejado, para que as perspectivas de produção possam ser condizentes.

As avançadas técnicas de determinação de parâmetros que avaliam a qualidade da madeira como, por exemplo, a densitometria de raios $\mathrm{X}$ usada na determinação da densidade aparente se constituem em importantes ferramentas numa caracterização mais detalhada do material, auxiliando em estudos que visem conhecer a formação do lenho das árvores, para recomendação do seu emprego tecnológico (BENJAMIN; BALLARIN, 2009).

Nesse contexto, o objetivo deste estudo foi avaliar a influência do espaçamento de plantio no crescimento em diâmetro e variação radial da densidade e das fibras da madeira de árvores de K. ivorensis.

\section{MATERIAL E MÉTODOS}

Local de plantio e amostragem: A espécie estudada foi a K. ivorensis A. Chev. proveniente de plantios experimentais da Atlântica Agropecuária, Pirapora, Norte do estado de Minas Gerais (latitude $17^{\circ} 21^{\prime} 55^{\prime \prime} \mathrm{S}$, e longitude $\left.44^{\circ} 56^{\prime} 59^{\prime \prime}\right)$. O clima é predominantemente tropical úmido (Aw) com estação chuvosa no verão e seca no inverno (classificação de Köppen), com precipitação e temperatura média anual de $1.057 \mathrm{~mm} \mathrm{e} 23,4^{\circ} \mathrm{C}$, respectivamente (INMET, 2014), e solo latossolo franco arenoso.

As árvores são oriundas de mudas de origem de sementes e o plantio das mesmas foi implantado em julho de 2008. Desde então vem sendo irrigado pelo sistema de micro aspersão, com uma média de 220 dias irrigados ao ano, cessando somente nos meses de dezembro a fevereiro devido à ocorrência de chuvas. Na Tabela 1, são descritas as características do plantio em função do espaçamento empregado.

Tabela 1. Descrição do plantio experimental de $K$. ivorensis aos cinco anos de idade.

Table 1. Description of the experimental planting Khaya ivorensis at five years of age.

\begin{tabular}{lcc}
\hline Espaçamento de plantio $(\mathbf{m})$ & Área da parcela $\left(\mathbf{m}^{2}\right)$ & Número de árvores/parcela \\
\hline $5 \times 5$ & 2.025 & 100 \\
$7 \times 6$ & 1.764 & 51 \\
$10 \times 10$ & 1600 & 24 \\
\hline
\end{tabular}

A coleta das amostras foi conduzida sob o Delineamento Inteiramente Casualizado (DIC), em que as repetições de cada parcela equivalem à quantidade de árvores amostradas. Foram selecionadas 10 árvores em cada parcela desconsiderando as árvores de bordadura. As amostras foram obtidas na forma de baguetas, coletadas pelo método não destrutivo com o uso de um extrator de 
madeira (Still BT- 45) a uma altura de 0,40 metros do solo, considerando as direções cardiais norte e sul de cada árvore, com objetivo de retirar amostras em direções opostas (duas amostras/árvore).

Crescimento em diâmetro: Foram obtidos dados referentes ao diâmetro a altura do peito - DAP (1,30 m do solo), das árvores por meio de medições utilizando-se uma suta. Com os dados de diâmetro das árvores, calculou-se o incremento médio anual das árvores, aplicando-se a equação 1.

$$
I M A D=\frac{D A P}{i d a d e}
$$

Em que:

IMAD = incremento médio anual em diâmetro $(\mathrm{cm} / \mathrm{ano})$

$\mathrm{DAP}=$ diâmetro à altura do peito $(\mathrm{cm})$

Idade $=$ idade das árvores (anos)

Densidade aparente da madeira: Foi determinada pela técnica de densitometria de raios X, cuja metodologia é descrita por Amaral e Tomazello Filho (1998).

As baguetas retiradas das posições norte e sul foram fixadas em suportes de madeira e seccionadas no sentido transversal, com espessura de 2,0 mm, utilizando-se uma dupla serra circular paralela. Posteriormente, as amostras foram acondicionadas em câmara de climatização por aproximadamente 12 horas, a uma temperatura de $20^{\circ} \mathrm{C}$ e umidade relativa em torno de $50 \%$, até atingir $12 \%$ de umidade.

A leitura das amostras foi realizada por um feixe colimado de raios X em intervalos de $80 \mu \mathrm{m}$ no equipamento QTRS-01X (Quintek Measurement Systems), sendo os dados de densidade aparente obtidos pelo software QMS, de acordo com os procedimentos empregados por Lobão et al. (2012).

Morfologia das fibras: O estudo morfológico das dimensões das fibras da madeira foi realizado seguindo as recomendações da Comissão Panamericana de Normas Técnicas (COPANT, 1974). Foram obtidas amostras do cerne periférico das baguetas extraídas da direção norte, como uma forma de padronização. Essas amostras foram reduzidas a pequenas lascas e maceradas em solução de ácido acético e peróxido de hidrogênio na proporção de 1:1. Os frascos contendo as amostras foram levados à estufa à temperatura de $60^{\circ} \mathrm{C}$ durante o período de 48 horas. A solução foi lavada com água destilada e as fibras foram coradas com safranina e preparadas lâminas histológicas utilizando a mistura de glicerina e água destilada na proporção de 1:1 (RAMALHO, 1987). Mediante a confecção das lâminas, foram realizadas fotomicrografias com auxílio de uma câmera fotográfica acoplada a um microscópio. As mensurações (20 por lâmina) do comprimento e espessura de parede das fibras foram realizadas por meio de um software analisador de imagens Image-Pro Plus (versão 4.5).

Análise dos dados: Para análise estatística dos dados utilizou-se o software R (versão 3.1.1). Os dados obtidos de DAP, IMAD, densidade aparente e dimensões das fibras foram submetidos ao teste de normalidade Shapiro-Wilk, e quando considerados com distribuição normal testou-se a homogeneidade das variâncias,sendo estes posteriormente submetidos à análise de variância (ANOVA), pelo teste $\mathrm{F}(\alpha=0,05)$, e quando esta foi significativa, as médias foram comparadas pelo teste de Tukey $(\alpha=0,05)$. A relação entre as variáveis DAP, densidade aparente e dimensões das fibras foi analisada por meio do coeficiente de correlação linear de Person e seu valor P. Em casos de correlação significativa, foram ajustados modelos lineares simples $(\mathrm{y}=\beta 0+\beta 1 \mathrm{x}+$ erro), sendo estes avaliados quanto ao $\mathrm{R}^{2}$ ajustado e o erro padrão da estimativa $\left(\mathrm{S}_{\mathrm{xy}}\right)$. Realizou-se também a análise de variância para os modelos de regressão (teste F) e o teste de $t$ para avaliar a significância de cada coeficiente estimado ao nível de 5\%.

\section{RESULTADOS E DISCUSSÃO}

Os valores de DAP e IMAD diferiram entre si em função do espaçamento de plantio (Figura 1A e B), indicando um maior crescimento nas árvores oriundas de espaçamentos de maior amplitude (7x6 e 10x10 m). Em média, os espaçamentos 7x6 e 10x10 m exibiram acréscimos diamétricos de 18,5\% e 20,5\%, respectivamente, em relação ao espaçamento 5x5 m. Conforme explicaram Lima et. al (2013), em espaçamentos maiores, é comum as árvores exibirem maior crescimento em diâmetro, pois há maior espaço para o crescimento individual das árvores, favorecendo o desenvolvimento da planta e promovendo maiores incrementos diamétricos ao longo dos anos. 

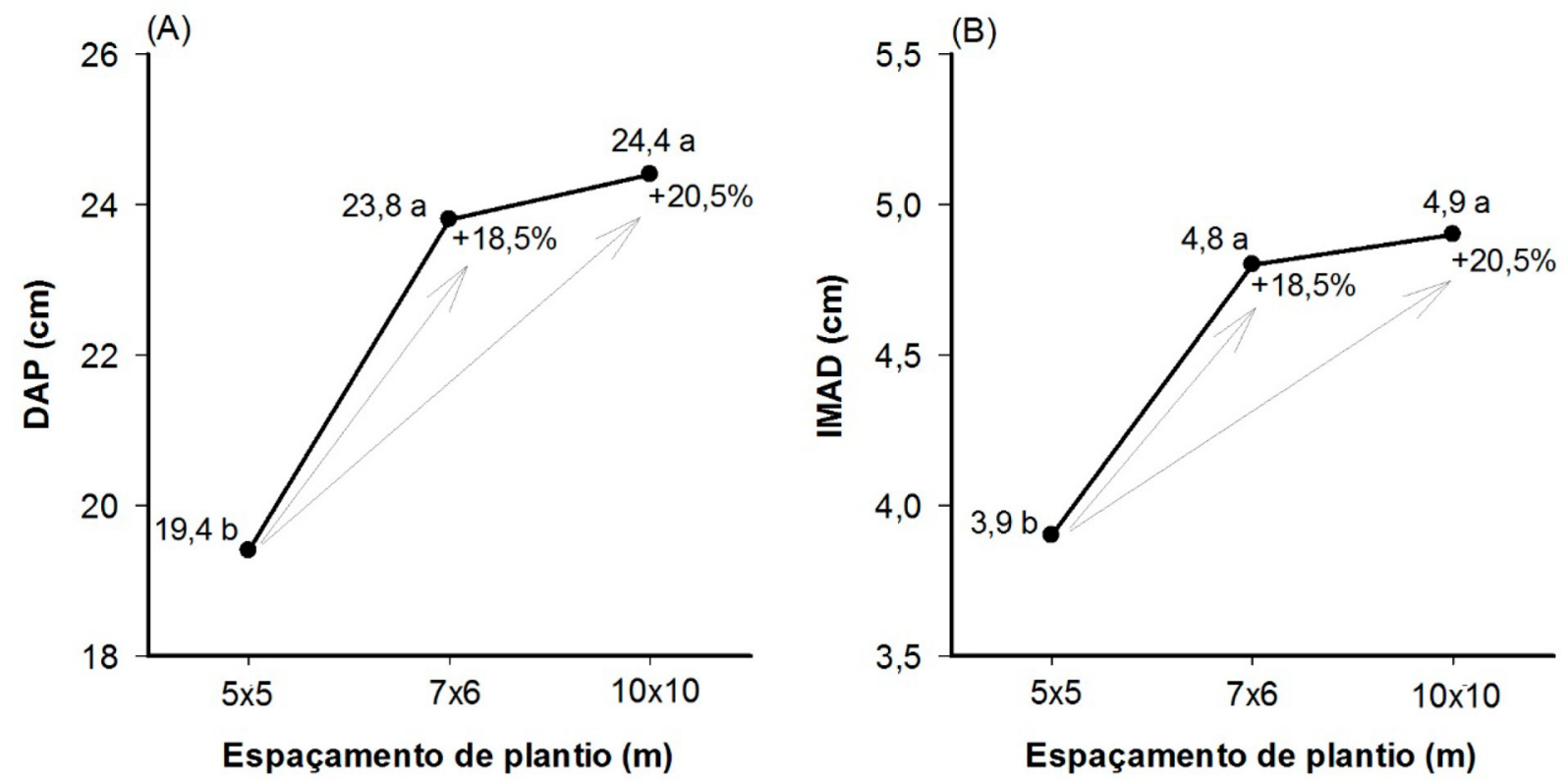

Figura 1. Valores médios de diâmetro a altura do peito (DAP) [A] e incremento médio anual em diâmetro (IMAD) [B] das árvores de Khaya ivorensis aos cincos anos de idade em função dos espaçamentos de plantio.

Figure 1. Mean diameter values at breast height (DBH) $[A]$ and mean annual increment in diameter (IMAD) $[B]$ of trees Khaya ivorensis at five years depending on planting space.

A tendência de crescimento em diâmetro proporcionado por espaçamentos de maior amplitude, também foi observada por autores para árvores de espécies de eucalipto. Exemplificando as pesquisas de Berger et al. (2002); Oliveira Neto et al. (2010) e Sereghetti et al. (2015) para Eucalyptus saligna (10 anos); E. camaldulensis (32 meses) e o híbrido de E. urophylla $x$ E. grandis (12 meses), respectivamente.

Dentre diversos fatores (espécie, produtos, forma do fuste, defeitos, maquinário e mão de obra), o rendimento em madeira serrada é influenciado principalmente, pelo diâmetro das toras (MURARA JR. et al., 2005). Neste sentido, em programas de manejo para a essa finalidade, é almejado que as árvores exibam satisfatório crescimento em diâmetro, conforme foi observado para os maiores espaçamentos (7x6 e 10x10 m).

Contudo, na situação de estudo, o espaçamento de plantio 7x6 m exibiu vantagens comparativas, pelo acréscimo em DAP e IMAD similar ao espaçamento de maior amplitude (10x10 m) e pelo maior volume de madeira/ha proporcionado. Ou seja, com a ampliação do espaçamento de plantio de 7x6 para 10x10 m, não há ganhos significativos no crescimento em diâmetro.

Em relação às características da madeira, verificou-se que a densidade aparente média e as dimensões das fibras na posição do cerne periférico da madeira não foram influenciados em função do espaçamento (Tabela 2). Ou seja, até a idade de cincos anos o espaçamento não exerceu efeito significativo nas propriedades do lenho das árvores de K. ivorensis avaliadas nesta pesquisa.

Tabela 2. Valores médios de densidade aparente e dimensões das fibras do cerne periférico do lenho de Khaya ivorensis aos cinco anos de idade em função do espaçamento de plantio.

Table 2. Mean values of density and fiber dimensions of the peripheral core of the wood of Khaya ivorensis at five years of age due to the planting spacing.

\begin{tabular}{lccc}
\hline Espaçamento de plantio $(\mathbf{m})$ & $\mathbf{D A}_{\mathbf{1 2 \%}}\left(\mathbf{g} \cdot \mathbf{c m}^{-3}\right)^{*}$ & $\mathbf{C F}(\boldsymbol{\mu m})$ & EP $(\boldsymbol{\mu m})$ \\
\hline $5 \times 5$ & $0,61 \mathbf{a}^{* *}$ & $1268,74 \mathbf{a}$ & $3,37 \mathbf{a}$ \\
$7 \times 6$ & $0,61 \mathbf{a}$ & $1200,62 \mathbf{a}$ & $3,62 \mathbf{a}$ \\
$10 \times 10$ & $0,59 \mathbf{a}$ & $1299,80 \mathbf{a}$ & $3,56 \mathbf{a}$ \\
\hline $\mathbf{C V}(\%)$ & $\mathbf{7 , 4 8}$ & $\mathbf{8 , 3 9}$ & $\mathbf{9 , 9 1}$ \\
\hline
\end{tabular}

*DA ${ }_{12 \%}$ : densidade aparente a $12 \%$ de umidade; $C F=$ comprimento das fibras; EP = espessura de parede, CV = coeficiente de variação. ***édia de uma mesma coluna seguida pela mesma letra não diferem entre si ao nível de $5 \%$ de probabilidade.

Em estudos realizados com a madeira de eucalipto em idade jovens, proveniente de diferentes áreas plantadas no Brasil, nenhum efeito significativo na densidade básica da madeira, foi observado por Sereghetti et al. (2015) e Moulin (2013) para o híbrido E. urophylla $x$ E. grandis, ambos, aos 12 meses de idade. 
Rocha (2011) também, não observou influência significativa do espaçamento na densidade básica da madeira de E. grandis $\mathrm{x}$ E. camadulensis, aos 48, 61 e 77 meses de idade, no entanto, verificou tendência de aumento desta propriedade conforme ampliação do espaçamento.

Já Feuchard (2015), verificou que o espaçamento influenciou de forma significativa a densidade básica da madeira, considerando o de menor e maior amplitude, observando, também a tendência de aumento da densidade básica da madeira, com o aumento da área vital de plantio para os híbridos de E. urophylla x E. grandis aos quatro e cincos anos de idade.

Contudo, nas idades próximas aos cinco anos de idade, observa-se que para a madeira de clones de híbridos de eucalipto existe uma tendência de aumento da densidade da madeira, com o aumento do espaçamento de plantio.

Porém, para a madeira K. ivorensis essa tendência não foi observada, visto que, os espaçamentos avaliados neste trabalho, exibem amplo espaço para desenvolvimento individual de uma planta, considerando os primeiros anos de crescimento. Assim, pode-se inferir que na idade de avaliação (cinco anos), a competição normalmente imposta entre indivíduos de um mesmo povoamento, ainda não tenha atingido intensidade suficiente ao ponto de alterar as características de formação da madeira.

Em relação às dimensões das fibras da madeira, Tienne et al. (2009) verificaram tendência de aumento dos valores do comprimento da fibra com a ampliação dos espaçamentos para madeira de um híbrido E. urophylla x E. grandis aos dois anos. Já, Moulin (2013), verificou situação inversa para a madeira do mesmo hibrido aos seis meses, notando também, que a espessura da parede tendeu ao aumento com o adensamento do espaçamento.

As principais fontes de variação da densidade e das dimensões das fibras da madeira em função do espaçamento são, de acordo com os autores supracitados, a espécie, o material genético (clone ou semente), características do sítio e idade, por exemplo. Em algumas espécies esses fatores podem exercem efeito de forma mais intensa na formação da madeira e já em outras espécies não, como foi observado para a madeira de K. ivorensis.

A realização de pesquisas nas fases iniciais de estabelecimento de florestas plantadas é uma forma de avaliar o desenvolvimento das plantas nas situações de crescimento impostas, visando subsídios ao melhoramento genético. Para as árvores de K. ivorensis, as propriedades da madeira avaliadas neste estudo, não demostraram ser influenciadas pelos diferentes espaçamentos avaliados. Dessa forma, pode-se inferir que até a idade de cincos anos a escolha do espaçamento de plantio para a espécie estudada levará em consideração os acréscimos no crescimento da planta, os quais distinguem-se em função do espaçamento.

As variáveis DAP e densidade aparente da madeira exibiram correlação significativa entre si $(\mathrm{r}=$ $-0,863 ; \mathrm{P}<0,05)$ no espaçamento de maior amplitude (10x10) [Figura 2]. Foi observado aumento dos valores de densidade nas árvores de menor DAP (Figura 2A), situação inversa ocorreu com o aumento do diâmetro (Figura 2B). Para os espaçamentos 5x5 e 7x6 m essas variáveis não correlacionaram entre si significativamente.

Garcia et al. (1991), também observaram tendência semelhante avaliando a influência dos espaçamento sobre a produtividade e as características da madeira de E. saligna e E. grandis aos 10 anos de idade. Conforme o aumento do DAP das árvores a densidade básica da madeira média diminuiu nos espaçamentos de maior amplitude.

O estudo de correlação entre as propriedades da madeira e características dendrométricas é importante, nos casos de relação significativa entre as variáveis, para propor modelos lineares que permitam a estimativa de uma determinada propriedade em função de outra de fácil e prática determinação ou mensuração. Nesse contexto, são marcantes as relações entre o DAP das árvores e as propriedades da madeira, uma vez que, o DAP é uma variável de fácil mensuração em campo.

Considerando que nesse estudo as variáveis DAP e densidade aparente da madeira das árvores cultivadas no espaçamento $10 \times 10 \mathrm{~m}$, apresentaram correlação significativa, foi possível propor um modelo linear, com $\mathrm{R}^{2}=0,71$, para estimar a DA em função do DAP, utilizando a equação 2 .

$$
\widehat{D A}=0,953-(0,0154 \times D A P)^{*}
$$

Em que:

$\mathrm{DA}=$ densidade aparente $\left(\mathrm{g} \cdot \mathrm{cm}^{-3}\right)$

$\mathrm{DAP}=$ diâmetro a altura do peito $(\mathrm{cm})$

Sci. For., Piracicaba, v. 44, n. 110, p. 519-526, jun. 2016 DOI: dx.doi.org/10.18671/scifor.v44n110.24 


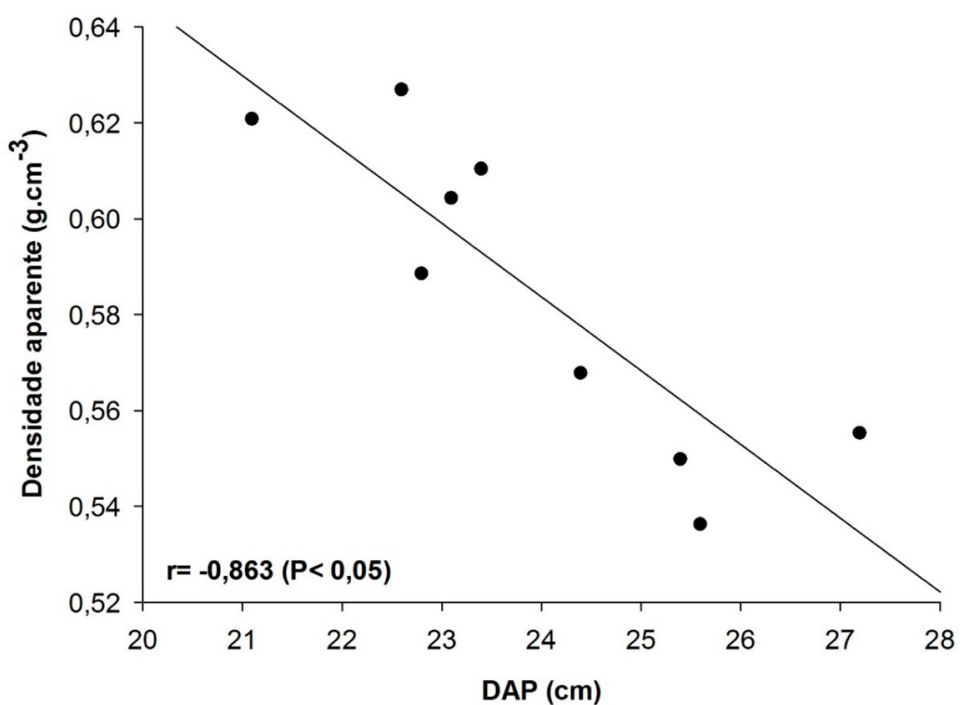

Figura 2. Diagrama de dispersão do diâmetro na altura do peito (DAP) e densidade aparente média da madeira de Khaya ivorensis aos cinco anos de idade, em espaçamento de plantio 10x10 metros.

Figure 2. Scatter diagram diameter at breast height $(\mathrm{DBH})$ and medium density wood of Khaya ivorensis at five years of age, planting spacing $10 \times 10$ meters.

A relação inversa observada entre as variáveis DAP e densidade aparente da madeira no espaçamento 10x10 m, é uma desvantagem para escolha deste tipo de espaçamento, aumentando a variabilidade da madeira produzida dentro de um mesmo povoamento.

Para a variação do perfil radial (medula-casca) da densidade aparente do lenho das árvores em função do espaçamento de plantio, observa-se uma tendência comum entre os espaçamentos de plantio 5x5, 7x6 e 10x10 m. Na região central da madeira, próximo à medula, a densidade aparente da madeira foi menor e conforme o distanciamento da medula tendeu a um aumento em maior variação nas últimas posições próxima à casca (Figura 3). O incremento radial nos primeiros anos (próximo à medula) exerce influência no decréscimo da densidade da madeira, pois as condições mais favoráveis ao rápido crescimento habitualmente conduzem a menores valores de densidade nessas regiões (KOUBAA et al., 2005).

Silva (2014), também observou tendência similar na variação da densidade aparente da madeira para espécie K. ivorensis cultivada em sistema de plantio em uma idade superior (19 anos).

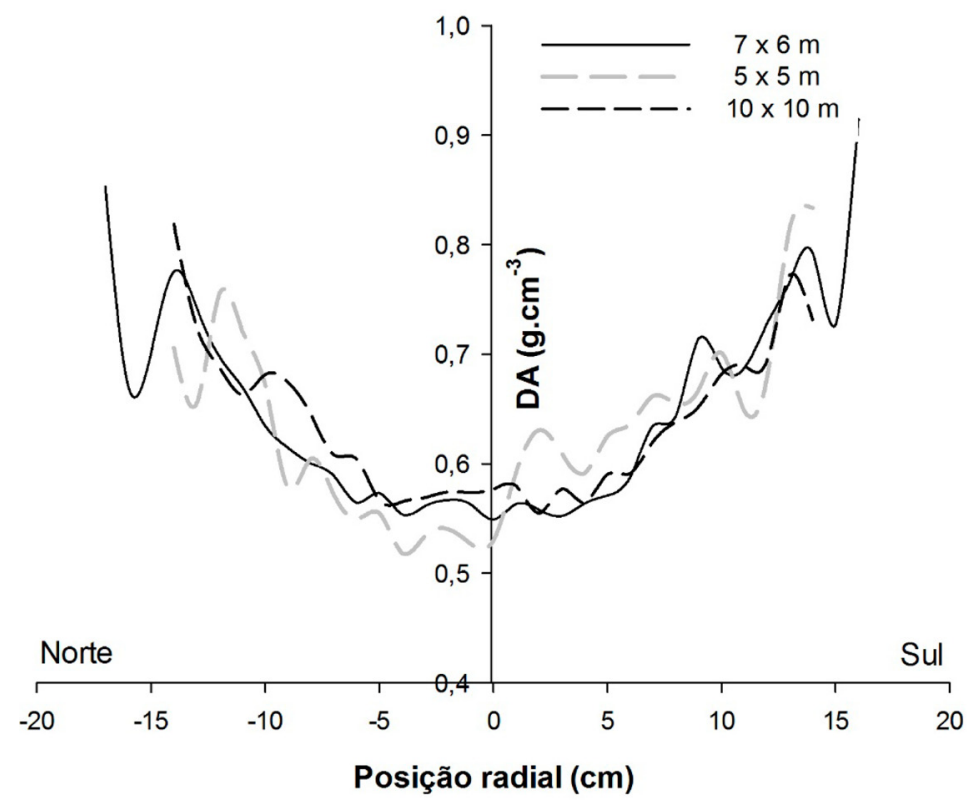

Figura 3. Variação radial (norte-sul) média da densidade aparente do lenho de Khaya ivorensis aos cinco anos de idade, em função dos espaçamento de plantio.

Figure 3. Radial variation (north-south) of the mean wood density of Khaya ivorensis wood at five years of age, depending on planting space. 
O aumento da densidade da madeira no sentido medula-casca está relacionado com a formação de madeira juvenil nos primeiros anos (SERPA et al., 2003; BALLARIN; LARA PALMA, 2003). Como o lenho de K. ivorensis avaliado é proveniente de plantios com idade de cinco anos, é conveniente que ocorra essa variação no perfil de densidade radial, pois a madeira formada até esse período é composta apenas por lenho juvenil.

\section{CONCLUSÕES}

A variação do espaçamento de plantio afeta significativamente o incremento médio radial, mas até determinados limites.

A densidade aparente da madeira e as dimensões das fibras do lenho de $K$. ivorensis até os cinco anos de idade não são afetadas pela variação do espaçamento de plantio;

No espaçamento $10 \times 10 \mathrm{~m}$, as árvores tenderam a DAP's menores e densidade da madeira superior a daquelas com maior diâmetro, implicando em uma variação da qualidade da madeira.

De forma geral, houve tendência comum no perfil de variação radial da densidade aparente para os três espaçamentos. Há um aumento da densidade da madeira no sentido medula-câmbio sem tendência de estagnação o que denota uma fase de formação de lenho exclusivamente jovem.

O espaçamento 7x6 m é o mais recomendado, pois apresenta crescimento em diâmetro superior ao espaçamento $5 \times 5$ m e comparável ao espaçamento 10x10 além de não apresentar variação na qualidade da madeira entre árvores.

\section{AGRADECIMENTOS}

A Atlântica Agropecuária pelo fornecimento do material para realização deste estudo, agradecimento especial ao Eng. Agrônomo João Emílio Martins por toda a logística para coleta de material.

A Fundação de Amparo à Pesquisa e Inovação do Espírito Santo - FAPES pelo financiamento do projeto de pesquisa e pela concessão da bolsa de mestrado.

\section{REFERÊNCIAS BIBLIOGRÁFICAS}

AMARAL, A. C. B.; TOMAZELlO FILHO, M. Avaliação das características dos anéis de crescimento de Pinus taeda L. segundo micro densitometria de Raios X. Revista de Ciência e Tecnologia, Piracicaba, v. 11, p. 17-23, 1998.

BALLARIN, A. W.; LARA PALMA, H. A. Propriedades de resistência e rigidez da madeira juvenil e adulta de Pinus Taeda L. Revista Árvore, Viçosa, v. 27, n. 3, p. 371-380, 2003.

BENJAMIN, C. A.; BALLARIN, A. W. Variação radial da densidade aparente da madeira de Corymbia (Eucalyptus) citriodora com 29 anos de idade. Energia na Agricultura, Botucatu, v. 24, n. 2, p. 29-46, 2009.

BERGER, R.; SCHNEIDER, P. R.; FINGER, C. A. G.; HASELEIN, C. R. Efeito do espaçamento e da adubação no crescimento de um clone de Eucalyptus saligna Smith. Ciência Florestal, Santa Maria, v. 12, n. 2, p. 75-87, 2002.

COPANT. COMISSÃO PANAMERICANA DE NORMAS TÉCNICAS. Método para la descripción de características generales, macroscópicas de las maderas angiospermas dicotiledóneas. Santiago, 1974. 19 p. (Informe, 30).

FERRAZ FILHO, A. C.; RIBEIRO, A. Inventário florestal do plantio de mogno africano (Khaya ivorensis A. Chev) e quadras experimentais. 4.ed. Pirapora: Fazenda Atlântica, 2013. 40 p.

FEUCHARD, L. D. Influência do espaçamento de plantio e idade de colheita na qualidade da madeira de eucalipto para celulose. 2015. 61 p. Dissertação (Mestrado em Ciências Florestais) - Universidade Federal do Espírito Santo, Jerônimo Monteiro, 2015.

Sci. For., Piracicaba, v. 44, n. 110, p. 519-526, jun. 2016 DOI: dx.doi.org/10.18671/scifor.v44n110.24 
Soranso et al. - Variabilidade física e anatômica da madeira de

Khaya ivorensis A. Chev. em diferentes espaçamentos de plantio

GARCIA, C. H.; CORRADINE, L.; ALVARENGA, S. F. Comportamento florestal do Eucalyptus grandis e Eucalyptus saligna em diferentes espaçamentos. IPEF, Piracicaba, 1991. 10 p. (Circular Técnica, 179).

INMET. INSTITUTO NACIONAL DE METEOROLOGIA. Normais climatológicas do Brasil 1961-1990. Disponível em: http://www.inmet.gov.br/portal/index.php?r=clima/normaisClimatologicas. Acesso em: 04 mai. 2014.

KOUBAA, A.; ISABEL, N.; ZHANG, S. Y.; BEAULIEU, J.; BOUSQUET, J. Transition from juvenile to mature wood in black spruce (Picea Mariana (MILL.) B.S.P.). Wood and Fiber Science, Hanover, v. 37, n. 3, p. 445455, 2005.

LEITE, H. G; NOGUEIRA, G. S.; MORREIRA, A. M. Efeito do espaçamento e da idade sobre variáveis de povoamentos de Pinus taeda L. Revista Árvore, Viçosa, v. 30, n. 4, p. 603-612, 2006.

LIMA, R.; INOUE, M. T.; FILHO, A. F.; ARAUJO, A. J.; MACHADO, S. A. Efeito do espaçamento no desenvolvimento volumétrico de Pinus taeda L. Floresta e Ambiente, Seropédica, v. 20, n. 2, p. 223-230, 2013.

LOBÃO, M. S.; COSTA, D. P.; ALMONACID, M. A. A.; TOMAZELlO FILHO, M. Qualidade do Lenho de árvores de Schizolobium parahyba VAR. amazonicum, Acre, Brasil. Floresta e Ambiente, Seropédica, v. 19, n. 3, p. 374-384, 2012.

MURARA JR., M. I.; ROCHA, M. P.; TIMOFEICZYK JR., R. Rendimento em madeira serrada de Pinus taeda para duas metodologias de desdobro. Floresta, Curitiba, v. 35, n. 3, p. 473-483, 2005.

MOULIN, J. C. Produção de madeira de eucalipto de curta rotação em povoamentos adensados para energia. 2013. 61 p. Dissertação (Mestrado em Ciências Florestais) - Universidade Federal do Espírito Santo, Jerônimo Monteiro, 2013.

OLIVEIRA NETO, S. N.; REIS, M. G. F.; LEITE, H. G.; NEVES, J. C. L. Crescimento e distribuição diamétrica de Eucalyptus camaldulensis em diferentes espaçamentos e níveis de adubação na região de cerrado de Minas Gerais. Floresta, Curitiba, v. 40, n. 4, p. 755-762, 2010.

RAMALHO, R. S. O uso de macerado no estudo anatômico de madeira. Viçosa: UFV, 1987 .4 p.

ROCHA, M. F. V. Influência do espaçamento e da idade na produtividade e propriedades da madeira de Eucalyptus grandis $\boldsymbol{x}$ Eucalyptus camaldulensis para energia. 2011. 71 p. Dissertação (Mestrado em Ciência Florestal). Universidade Federal de Viçosa, Viçosa, 2011.

SEREGHETTI, G. C.; LANÇAS, K. P.; SARTORI, M. S.; REZENDE, M. A.; SOLER, R. R. Efeito do espaçamento no crescimento e na densidade básica da madeira de Eucalyptus urophylla X Eucalyptus grandis em florestas de ciclo curto. Energia na Agricultura, Botucatu, v. 30, n. 3, p. 257-262, 2015.

SERPA, P. N.; VITAL, B. R.; DELLA LUCIA, R. M.; PIMENTA, A. S. Avaliação de algumas propriedades da madeira de Eucalyptus grandis, Eucalyptus saligna e Pinus elliottii. Revista Árvore, Viçosa, v. 27, n. 5, p. 723-33, 2003.

SILVA, J. G. M. Qualidade da madeira serrada e demarcação do lenho juvenil e adulto do mogno africano. 2014. 53 p. Dissertação (Mestrado em Ciências Florestais) - Universidade Federal do Espírito Santo, Jerônimo Monteiro, 2014.

SIMÕES, J. W.; BRANDI, R. M.; MALINOVSKY, J. R. Formação de florestas com espécies de rápido crescimento. Brasília: PNUD/FAO/IBDF/BRA, 1976. 74 p. (Série divulgação, 45,6).

TIENNE, D. L. C.; OLIVEIRA, J. N.; PALERMO, G. P. M.; SOUSA, J. S.; LATORRACA, J. V. F. Influência do espaçamento no ângulo das microfibrilas e comprimento de fibras de clone de eucalipto. Revista Forestal Latino americana, Mérida, v. 24, n. 1, p. 67-83, 2009.

Recebido em 15/05/2015

Aceito para publicação em 04/12/2015 